\title{
Sulphur K-edge XANES spectroscopy of transthyretin amyloid fibres
}

\author{
L. Gales ${ }^{\text {a,b }}$, I. Cardoso a,c, B. Fayard ${ }^{\text {d }}$, M.J. Saraiva ${ }^{\text {a,c }}$ and A.M. Damas ${ }^{\text {a,b,* }}$ \\ ${ }^{a}$ ICBAS, Instituto de Ciências Biomédicas Abel Salazar, Universidade do Porto, Largo Prof. Abel \\ Salazar no 2, 4099-003 Porto, Portugal \\ ${ }^{\mathrm{b}}$ Grupo de Estrutura Molecular, Instituto de Biologia Molecular e Celular, Universidade do Porto, \\ rua do Campo Alegre $n^{\circ} 823,4150$ Porto, Portugal \\ ${ }^{\mathrm{c}}$ Grupo de Amilóide, Instituto de Biologia Molecular e Celular, Universidade do Porto, rua do Campo \\ Alegre $n^{0} 823,4150$ Porto, Portugal \\ ${ }^{\mathrm{d}}$ European Synchrotron Radiation Facility (ESRF), BP 220, F-38043 Grenoble Cedex, France
}

\begin{abstract}
Transthyretin (TTR) amyloidosis results from the self-association of TTR leading to insoluble deposits that occur at the extra cellular level. Sulphur K-edge XANES spectroscopy was used to determine the sulphur oxidation state in transtyretin present in amyloid fibrils. These data is discussed in relation to molecular mechanisms involved in amyloid fibril formation.
\end{abstract}

\section{Introduction}

Transthyretin (TTR) is a plasma protein implicated in the transport of thyroxine and vitamin A. It is a homotetramer, with an extensive $\beta$-sheet structure. Each monomer has two four-stranded $\beta$-sheets and the four monomers associate through hydrogen bonds and hydrophobic interactions around a well defined channel where is located the thyroxine binding site. More than 80 TTR different variants are known [1], most of them are pathogenic and related to amyloidosis. The packing interactions of the protein subunits in TTR fibres, namely the possible role of sulphur-containing amino acids, remain unknown. Based on structural and biochemical analysis of the most frequent amyloidogenic variant, V30M-TTR, it has been proposed that amyloid fibrils could result from association of TTR molecules through disulphide bridges [2,3].

Sulphur K-edge X-ray absorption spectroscopy provides an analytical tool for the analysis of biological materials without chemical pre-treatment and consequently without perturbation of the sulphur redox state [4]. Here we present the spectra of V30M-TTR fibrils and of some reference compounds.

\section{Experimental}

The reference compounds cysteine, cystine, methionine, methionine sulfoxide, and anthraquinone-2sulfonic acid were purchased from Sigma and used without further purification.

V30M-TTR mutant was produced in an Escherichia coli expression system, isolated and purified as described elsewhere [5]. Amyloid fibrils were formed by acidification as previously described [6].

\footnotetext{
*Corresponding author. Tel.: +351 226074900; Fax: +351 226099157; E-mail: amdamas@ibmc.up.pt.
} 
Sulphur K-edge spectra were recorded at room temperature using synchrotron radiation, at the European Synchrotron Radiation Facility (ESRF), beam line ID21. The reference compounds were analysed in the form of powder and in solution and no differences were observed between the corresponding spectra. Fibril solutions were left to sediment for a few hours and a fraction of the pellet was used to record the spectra.

\section{Results and discussion}

The K-edge spectra of cysteine, cystine, methionine, methionine sulfoxide and anthraquinone-2sulfonic acid are shown in Fig. 1. The energies of maximum absorption for these compounds are 2.4730, $2.4723,2.4731,2.4759$ and $2.4809 \mathrm{keV}$, respectively. These values are in agreement with those described in previous studies [4,7]; the shapes of the spectra are clearly different except for cysteine and methionine that look very similar.

The K-edge spectrum of V30M-TTR fibrils is shown in Fig. 2. The V30M-TTR is a tetramer with subunits composed of identical 127 amino acids. Each monomer has one cystein and two methionines. Comparison with the reference spectra indicates the presence of cysteine and/or methionine, methionine sulfoxide and sulfonated cystein. To quantify the relative amount of each sulphur form, the protein spectrum was simulated by linear combination of the spectra of the reference samples. The best fit was obtained with $50 \%$ of cysteine and/or methionine, $19 \%$ of methionine sulfoxide and $31 \%$ of sulfonated cystein. Therefore there is a significant oxidation of sulphur in the fibrils, especially of cystein since $33 \%$ of the total amount of sulphur belongs to the cystein residue being $31 \%$ sulfonated. In relation to the two methionine residues, which make up $67 \%$ of the total sulphur in the sample, only $19 \%$ are oxidised. The X-ray crystallographic structure of variant V30M-TTR has been determined [2] and shows that one of the methionines, Met30, is buried in the molecule and not exposed to the solvent. Therefore, it is conceivable that only the other methionine, Met13, is exposed to the solvent and oxidised.

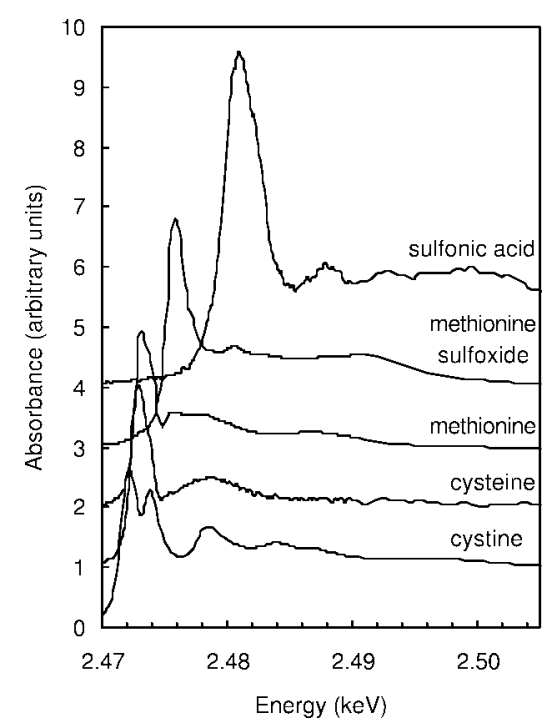

Fig. 1. Sulphur K-edge XANES spectra for some reference compounds. 


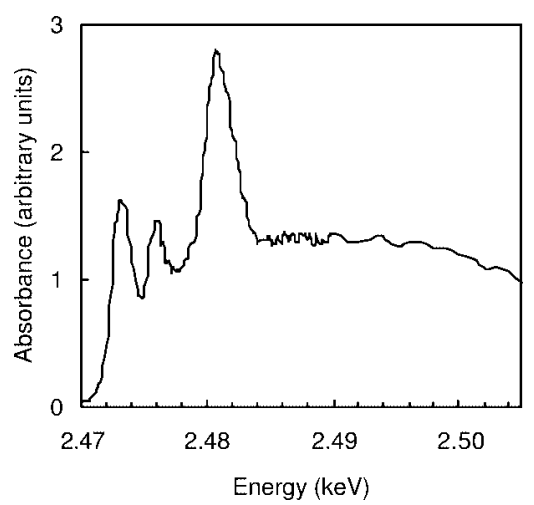

Fig. 2. Sulphur K-edge XANES spectrum of V30M-TTR fibrils.

\section{Conclusions}

There is a significant oxidation of sulphur in cystein and a partial oxidation of sulphur in methionines in V30M-TTR amyloid fibrils. Possibly this results from a conformational alteration of the protein during or upon polymerisation, exposing these residues to the solvent.

Further studies considering other amyloidogenic variants will give us insight into the molecular packing of proteins in amyloid fibrils.

\section{Acknowledgements}

We thank the Fundação para a Ciência e Tecnologia for financial suport (projects POCTI-35735/99 and POCTI/SAU/14095/1998). We acknowledge use of beam line ID21 of the European Synchrotron Radiation Facility (ESRF). We are also grateful for the technical assistance of the ESRF staff.

\section{References}

[1] M.J.M. Saraiva, Human Mutation 17 (2001), 493-503.

[2] C. Terry, A.M. Damas, P. Oliveira, M.J. Saraiva, I. Alves, P. Costa, Y. Sakaki and C. Blake, EMBO J. 12 (1993), $735-741$.

[3] C. Thylen, J. Wahlqvist, E. Haettner, O. Sandgren, G. Holmgren and E. Lungren, EMBO J. 12 (1993), 743-748.

[4] A. Rompel, R.M. Cinco, M.J. Latimer, A.E. McDermott, R.D. Guiles, A. Quintanilha, R.M. Krauss, K. Sauer, V.K. Yachandra and M. Klein, Proc. Natl. Acad. Sci. USA 95 (1998), 6122-6127.

[5] H. Furuya, M.J. Saraiva, M.A. Gawinowicz, I.L. Alves, P.P. Costa, H. Sasaki, I. Goto and Y. Skaki, Biochemistry 30 (1991), $2415-2421$.

[6] I. Cardoso, C.S. Goldsbury, S.A. Muller, V. Olivieri, S. Wirtz, A.M. Damas, U. Aebi and M.J. Saraiva, J. Mol. Biol. 317 (2002), 683-695.

[7] G. Sarret, J. Connan, M. Kasrai, L. Eybert-Bérard and G.M. Bancroft, J. Synchrotron Rad. 6 (1999), 670-672. 


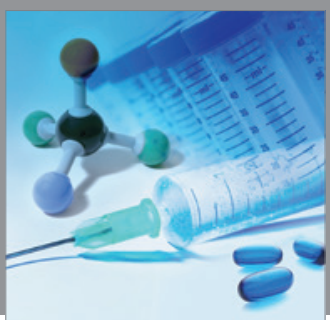

International Journal of

Medicinal Chemistry

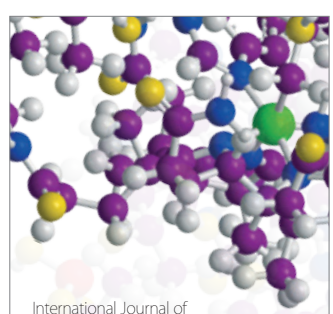

Carbohydrate Chemistry

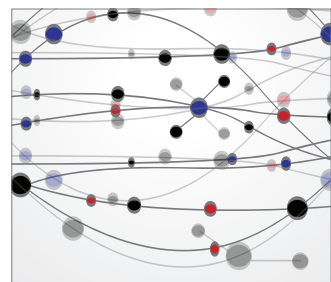

The Scientific World Journal
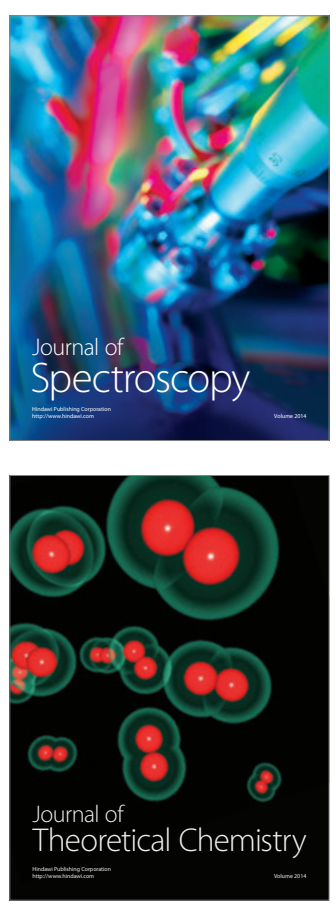
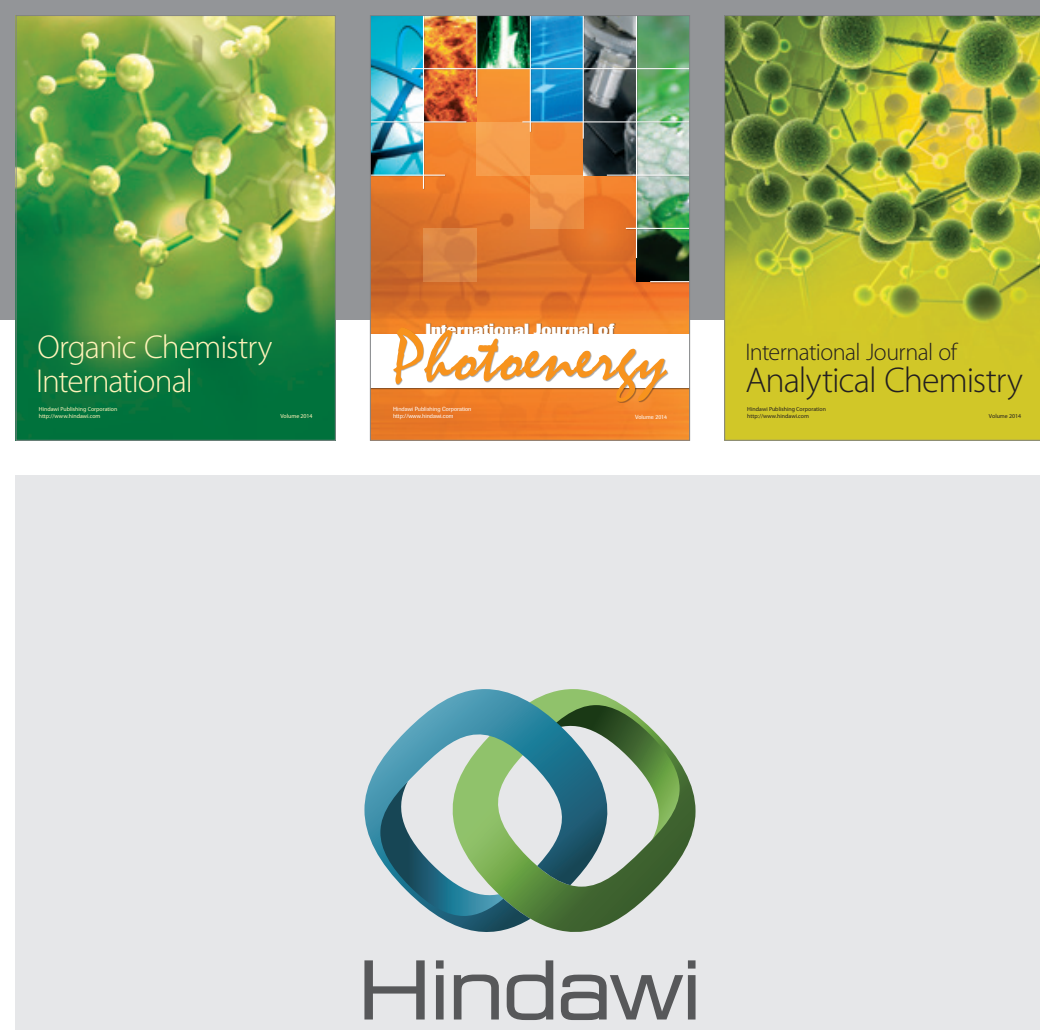

Submit your manuscripts at

http://www.hindawi.com
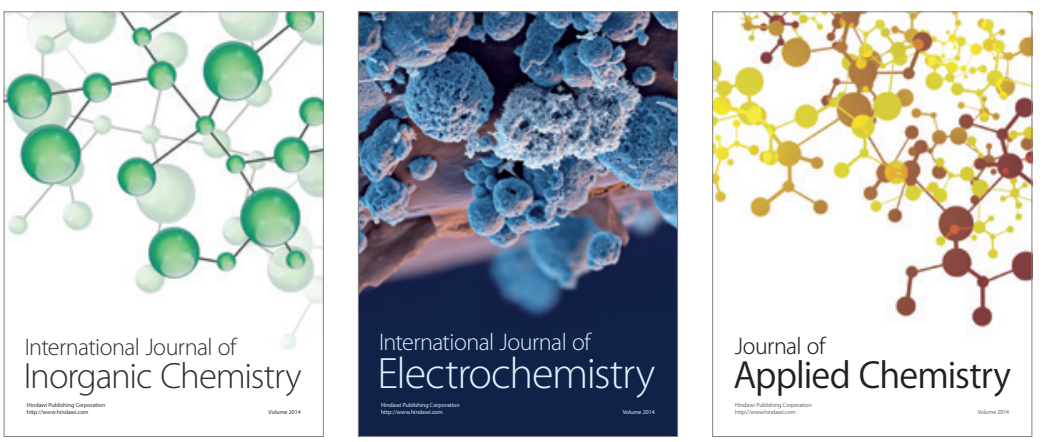

Journal of

Applied Chemistry
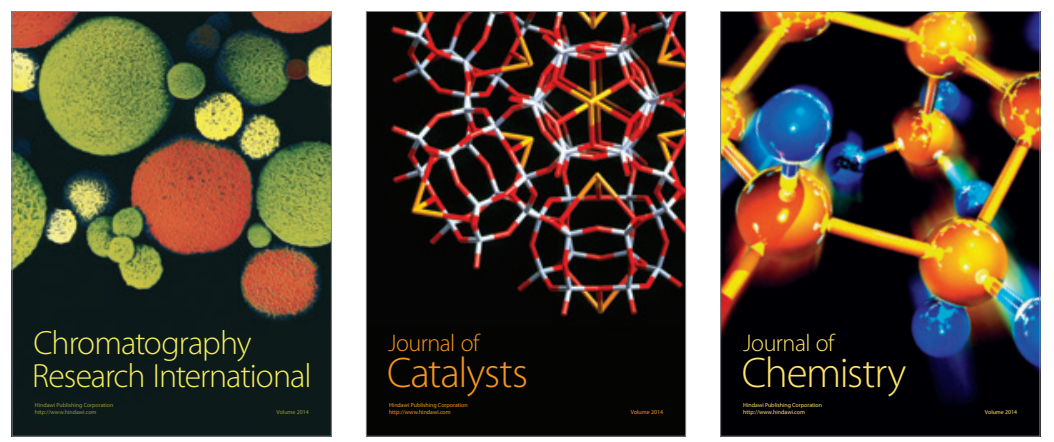
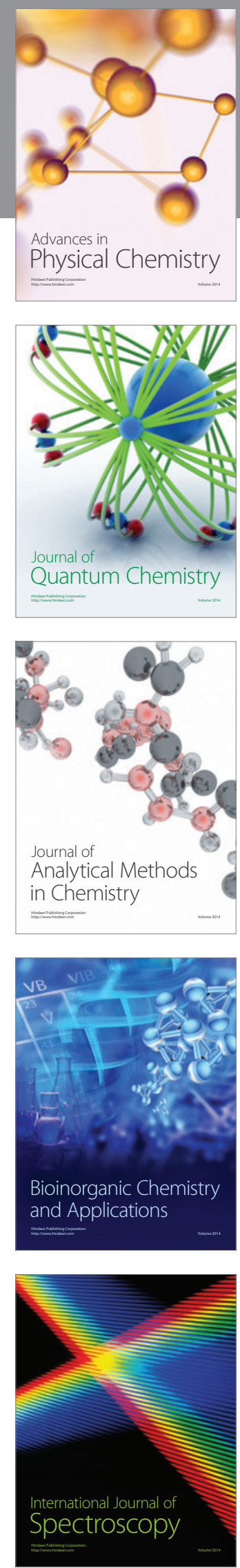